\title{
El servicio comunitario, un proyecto de innovación pedagógica
}

\author{
Gené Gordó i Aubarell \\ Departamento de Educación, Generalitat de Catalunya
}

\section{Resumen}

Formar personas completas, con una dimensión tan intelectual como cívica y moral que les permita ser competitivas en el mundo del trabajo, pero también capaces de analizar el entorno en que viven y de comprometerse para mejorarlo -lo que denominamos la ciudadanía activa- constituye la finalidad educativa. Ese es el objetivo del servicio comunitario impulsado por el Departamento de Educación, una acción educativa en el marco de la enseñanza obligatoria. El servicio comunitario nace con vocación innovadora, responde a una necesidad, situar la competencia social y ciudadana al mismo nivel que el resto de las competencias básicas, y para ello plantea provocar un cambio de mejora y cualidad educativa. El artículo ofrece una descripción de las diferentes fases de desarrollo del proyecto y aporta algunos datos de interés sobre su impacto.

\section{Palabras clave}

Aprendizaje-servicio, servicio comunitario, innovación pedagógica, política educativa. 


\title{
Community Service, a pedagogical innovation project
}

\begin{abstract}
The ultimate goal of education is to shape all-round professionals, those with an intellectual but also moral and civic dimension that would enable them not only to compete in the professional world, but also to analyse the environment in which they live and engage meaningfully in its betterment -this is what is known as active citizenship. This is precisely the objective of an educational initiative, promoted by the Department of Education of Catalonia, that has been integrated into compulsory education: the Community Service. The Community Service arose as an innovative action in response to a specific need: the need to confer the same worth to social and civic competencies as the rest of the basic competencies. To that aim, Community Service seeks to bring about a transformational change for the improvement of educational quality. This article aims to describe the project's various stages of development and to provide relevant information about its impact.
\end{abstract}

\section{Keywords}

Service-learning, community service, pedagogical innovation, educational policy. 
Lo que nos hace ciudadanos hoy es nuestra capacidad de compromiso social. La vida democrática viva y renovada...depende que todos tengamos el coraje de querer ser ciudadanos

Josep-Maria Terricabras

Hace tiempo que el término compromiso social transita en la agenda educativa internacional. Los resultados de algunos estudios nos alertan de que, a pesar de que existe una ligera tendencia a aumentar entre los jóvenes aquellos valores asociados a una dimensión social y comunitaria, son los valores hedonistas y del bienestar los que claramente prevalecen hoy (Jóvenes y valores, 2014). Una tendencia social orientada a cubrir y crear necesidades de realización y bienestar personal sin duda ha alimentado esta actitud. Pero cabe preguntarse si no ha contribuido también el impulso de ciertas prácticas educativas más centradas en los derechos que en los deberes, rompiendo ese equilibrio necesario que define la ciudadanía y potenciando un posicionamiento más cercano al del cliente exigente que al del ciudadano responsable y comprometido.

Algunos expertos consideran que esa tendencia social está directamente relacionada con la forma de entender y ejercer la propia ciudadanía. Hoy concebimos la ciudadanía principalmente como una obtención de derechos, relegando a menudo, a las personas elegidas democráticamente, la actividad ciudadana. Perdemos en ese movimiento la posibilidad de entender la ciudadanía como experiencia viva o dicho de otra forma, como capacidad humana. "Ser ciudadano tiene que ver con la voluntad de coger el destino con las propias manos y de hacer con ello alguna cosa". (Terricabras, 2008, pág.45).

Es importante resaltar que el marco normativo educativo expresa la necesidad de avanzar hacia esa capacidad de ciudadanía activa cuando, por ejemplo, formula la competencia social y ciudadana como aquella que hace posible comprender la realidad social en que se vive y comprometerse a contribuir en su mejora. Pero algo pasa cuando, a pesar de ello -según el estudio anteriormente mencionadoobservamos que mientras un $57 \%$ de jóvenes proclaman valores como la solidaridad, tan solo un $6 \%$ declaran, por ejemplo, comprometerse activamente en el barrio o la población en que viven. Es decir, parece ser que tenemos jóvenes muy comprometidos con valores finalistas como la justicia o la igualdad, pero no tanto con aquellos otros valores instrumentales (compromiso, esfuerzo, implicación...) sin los cuales los primeros "no pasan de ser cantos al sol" (Elzo, 2007).

Tenemos que reforzar esa idea de que la finalidad educativa es formar personas completas, con una dimensión tan intelectual como cívica y moral que les permita ser competitivas en el mundo del trabajo, sí, pero también capaces de analizar el entorno en que viven y de comprometerse para mejorarlo. Entre todos tenemos que ser capaces de evitar que nuestros jóvenes queden sumergidos en la llamada cultura del yo. Debemos despertar en ellos el deseo de poner sus capacidades 
y conocimientos al servició de los demás y comprometerse, de forma crítica, en la mejora de nuestra sociedad. En otras palabras, formarlos en lo que denominamos la ciudadanía activa.

Algunos países como Estados Unidos, Holanda o Argentina han reaccionado ante esta situación y han desarrollado acciones de compromiso cívico como parte de su formación obligatoria. Sensibles a esta necesidad formativa, el Departamento de Educación de la Generalitat de Catalunya impulsa el servicio comunitario, una acción en el marco de la enseñanza obligatoria orientada a adquirir y desarrollar la competencia social y ciudadana. Trabajando sobre necesidades reales con entidades sociales del tercer sector -sea en acciones dirigidas a las personas mayores, la preservación del medio o el apoyo a las necesidades básicas-, los jóvenes analizan el contexto y ponen su conocimiento y esfuerzo al servicio de la comunidad, a la vez que van adquiriendo -en esa acción- los valores propios de la competencia social y ciudadana.

\section{El servicio comunitario, un planteamiento innovador}

El servicio comunitario responde por tanto, a una necesidad social, formar ciudadanos implicados y comprometidos con su sociedad. Para ello se plantea provocar un cambio de mejora y cualidad educativa, situar la competencia social y ciudadana al mismo nivel que el resto de las competencias y crear una acción educativa eficaz para desarrollarla. Cambio y mejora son dos palabras incluidas en toda definición de innovación, entre ellas la del propio Departamento de Educación que la considera como un proceso planificado de cambio y renovación que se fundamenta en la investigación, que responde a la evolución social y que conduce a obtener una mejora en la calidad del sistema educativo.

Ciñéndonos a esa definición, podríamos considerar que el servicio comunitario nace con vocación innovadora, y a nuestro entender se consolida, como tal, al reunir las siguientes condiciones:

- Reconocimiento del problema e identificación de las formas de resolverlo

- Toma de decisiones

- Concreción de la propuesta

- Pilotaje

- Análisis de los riesgos y corroboración de la propuesta

1.1. Reconocimiento del problema e identificación de las formas de resolverlo

El servicio comunitario nace de la necesidad formar ciudadanos activos, implicados y comprometidos en la mejora y transformación social. Es oportuno mencionar que el servicio comunitario cuenta con un bagaje de experiencias previas sumamente valiosas. De entrada cabe subrayar su raíz identitaria. Cataluña proviene de una larga tradición de participación cívica y dispone de un fuerte tejido asociativo formado por personas que dedican su tiempo y esfuerzo al servicio de los demás. Pero también en el 
ámbito educativo son muchos los centros escolares que, bajo el nombre de aprendizaje-servicio u otras denominaciones, han ido desarrollando buenas prácticas en materia de servicio a la comunidad. Es importante destacar el impulso que desde 2005 viene llevando a cabo el Centro Promotor de Aprendizaje-Servicio. También el propio Departamento, en el marco de los Planes educativos de entorno, está promocionado desde 2008 este tipo de acciones educativas. Por tanto, coherente con esa herencia educativa, no es de extrañar que optáramos por crear un proyecto, el servicio comunitario mediante una metodología de aprendizaje-servicio que nos permitía adquirir des de la práctica la competencia social y ciudadana. Una propuesta educativa, que "vincula éxito educativo con el compromiso cívico. No puede haber un buen aprendizaje de competencia sin compromiso cívico, pero el compromiso cívico tampoco será de calidad si se desvincula del conocimiento" (Puig, 2009, 11).

\subsection{Toma de decisiones}

Como ya se ha comentado, contamos con muchas y buenas experiencias, pero también cabe mencionar que, a menudo, esas acciones de servicio a la comunidad dependen demasiado de la buena voluntad de algunos docentes y pocas veces, están vinculadas con el proyecto educativo de centro. Este hecho dificulta que las propuestas se lleguen a consolidar y mucho menos que generen una innovación. Estas experiencias, en general, no llegan a afectar a la estructura del centro ni sus dinámicas educativas (espacios de coordinación estables, vinculación con el currículo, evaluación...). Por tanto, nos preguntamos si ese efecto contagio, propio de las dinámicas de impulso y sensibilización, basado en la voluntad de algunos docentes, era suficiente para conseguir nuestro objetivo. Por otra parte, también nos hicimos una reflexión; si realmente creíamos que la educación era una herramienta orientada a formar seres con una dimensión tanto intelectual como humana y moral, que teníamos que ir avanzando -junto con las competencias lógico-matemática, lingüística o comunicativa- también en aquellas competencias que recogían la educación en valores y ciertas virtudes cívicas; si realmente creíamos que educar era también formar a jóvenes capaces de comprometerse con su sociedad, ¿no era ese un deber que teníamos para todo el alumnado? Y por lo tanto, ¿no debía formar parte del currículo obligatorio?

La respuesta nos pareció evidente. Por tanto, después de analizar la experiencia de aquellos países que con diferentes nominaciones, Servicelearning, Civic Intership, o Servicio sociocomunitario Solidario, estaban desarrollado políticas educativas similares, decidimos situar el servicio comunitario como parte del currículo obligatorio con la finalidad de garantizar que los estudiantes, a lo largo de su trayectoria escolar, experimentasen y protagonizasen acciones de compromiso cívico al mismo tiempo que, con esa acción, adquirían aquellas habilidades y capacidades propias de la competencia social y ciudadana. Y así se recogió en el DECRETO 187/2015, de 25 de 
agosto, de ordenación de las enseñanzas de la educación secundaria obligatoria.

Cabe resaltar que esa decisión también respondía a una necesidad normativa. La Ley 12/2009, del 10 de julio, de Educación de Cataluña, en su artículo 79.1.e) establece que los criterios de organización pedagógica que adopten los centros en las etapas que integran la educación básica deben contribuir a educar a los alumnos en la responsabilidad de ejercer la ciudadanía activa a través de la participación en los asuntos de la comunidad. En este sentido, el servicio comunitario puede entenderse como un desplegamiento de la propia Ley de Educación. Y eso es relevante porque la innovación pedagógica, al mismo tiempo que debe responder a una demanda social objetiva, ha de dar respuesta también a la normativa vigente y ser compatible con ella, garantizando así la coherencia con la línea educativa de país y facilitando también, la aplicación de esa innovación.

\subsection{Concreción de la propuesta}

Ya hemos comentado que el primer objetivo de la propuesta era desarrollar la competencia social y ciudadana, favoreciendo la actitud crítica y comprometida de nuestros jóvenes ante la sociedad. Un segundo objetivo que nos planteamos fue aumentar la participación del alumnado en el tejido asociativo. Con ello queríamos promover el sentimiento de pertenencia de nuestros alumnos, especialmente aquellos más vulnerables, mediante su arraigo en el territorio. Además, la participación en estas entidades permitía generar espacios de interacción y convivencia, creando entornos más cohesionados. Por otra parte, entendíamos que la participación de los jóvenes en las diferentes entidades podía facilitar, posteriormente, que algunos de ellos quisieran seguir con ese vínculo, aumentando así, el capital social de un territorio y por tanto, su capacidad de trasformación. Potenciar el voluntariado y mejorar el entorno son también objetivos del servicio comunitario.

Todos esos objetivos nos llevaron a replantear los procesos de aprendizaje. Necesitábamos una metodología que nos permitiera ese doble juego, comprometer al alumnado con su entorno inmediato al mismo tiempo que en esa acción adquiría las habilidades y capacidades propias de la competencia social y ciudadana. En ese sentido, la metodología aprendizaje-servicio nos permitía no solo educar para la vida sino para transformar la vida. El aprendizaje-servicio "sitúa a los jóvenes no solo en el centro de la educación sino también en el centro de la sociedad" (Martín, 2009, 111). El servicio comunitario entiende el alumno como un motor de cambio, capaz de ejercer la ciudadanía de forma activa desde su propia formación.

Por otra parte, situar el servicio comunitario como parte del currículum obligatorio significaba tener incidencia en la cualificación global de la materia o materias a la cual estuviera vinculada. Profundizar en la evaluación del alumnado era un requisito. Es necesario que el alumnado se convierta en competente, es decir, que tenga la 
capacidad de aplicar los conocimientos y las habilidades aprendidas para resolver problemas. Por tanto concretamos que habilidades, valores y actitudes requerían esa competencia, como desarrollarlas y que indicadores utilizar para evaluarlas.

\subsection{Pilotaje}

Una vez tomada la decisión y concretado el proyecto, y antes de iniciar la fase de difusión e implementación, era necesario validar la propuesta. Pilotamos la experiencia en 89 centros educativos. En el curso 2012/2013 se realizó la formación. Para ello contamos con la ayuda inestimable del Grupo Promotor de AprendizajeServicio. Esos centros implementaron durante el curso siguiente 118 proyectos en el que participaron 139 entidades socioeducativas.

El balance evaluativo fue muy positivo. El $97 \%$ de los centros educativos consideraron que el servicio comunitario contribuía notablemente a la adquisición de los valores y actitudes propias de la competencia social y ciudadana, objetivo principal del proyecto. Este dato parecía corroborar que los valores se aprenden ejerciéndolos, que educar para la ciudadanía exige superar el marco academicista del trabajo en el aula. Así mismo, el $69 \%$ observaba una notable mejora de los resultados académicos en la materia o materias en las cuales se había desarrollado el servicio comunitario, hecho que reforzaba aquella idea de que los jóvenes mejoran sus resultados académicos "al ver la utilidad social de aquello que aprenden, y sentirse reconocidos y valorados" (Batlle, 2013, 26). También hay que resaltar que un $23 \%$ del alumnado manifestó su interés por seguir colaborando con las entidades una vez finalizado el proyecto, dato importante dado que uno de los objetivos era aumentar el asociacionismo y la participación de nuestros jóvenes con las entidades del entorno.

\subsection{Análisis de los riesgos y corroboración de la propuesta}

Situar el servicio comunitario en el marco curricular obligatorio conllevaba algunos riesgos que era preciso evitar. Por una parte, la posible resistencia que podía suscitar en el profesorado y el propio alumnado una acción no voluntaria y por otra parte, la posible banalización del proyecto, reduciéndolo, por ejemplo, a un mero asistencialismo o bien a una acción puramente de crítica social, prácticas ambas- claramente deficitarias. Esos riegos nos fueron planteados por varios de nuestros colaboradores. Pero dado que la necesidad y bondad del proyecto no era cuestionado en ninguno de los casos, decidimos que lo oportuno no era replantear la obligatoriedad del proyecto, sino evitar los riegos que este originaba.

Debíamos poner un énfasis especial en la formación y sensibilización, pero también debíamos generar modelos transferibles, es decir ejemplos de servicio comunitario con actividades didácticas y recursos que marcaran sistemáticamente todas las fases del proyecto (sensibilización, análisis crítico, propuesta y realización del servició y autoevaluación). El objetivo 
era evitar las posibles desviaciones que antes comentábamos y la banalización del proyecto.

Una vez evaluado el pilotaje y diseñados los instrumentos necesarios para evitar los posibles riesgos, se corroboró la decisión e introducimos el servicio comunitario en el marco normativo curricular como "una acción educativa de carácter obligatorio orientada a desarrollar la competencia social y ciudadana, en que el alumno, con el fin de mejorar su entorno, realiza un servicio a la comunidad, aplicando sus conocimientos, capacidades y habilidades, a la vez que aprende el ejercicio activo de la ciudadanía". (DECRETO 187/2015, art16). Llegados ahí, se planificó la difusión e implementación del proyecto.

\section{Difusión e implementación del servicio comunitario}

Seguidamente intentaremos explicar la fase de difusión atendiendo a las acciones que lleva asociada toda innovación.

\subsection{Sensibilización y dinamización de todos los implicados}

En ese sentido, era indispensable tejer complicidades con las principales entidades culturales y sociales, como partner imprescindibles: son estas entidades las que facilitan a los centros educativos, des de su actividad cotidiana, el espacio para desarrollar el servicio comunitario. Era oportuno reforzar el concepto win-win del proyecto, remarcando la dimensión educativa de la actividad de estas entidades y también el beneficio que el servicio comunitario podía proporcionarles, creando la posibilidad de incrementar sus bases, a partir de la participación de los jóvenes. Contamos desde un primer momento con el apoyo de diferentes colectivos, entre otros, la Mesaa del Tercer Sector o Lafede, con quienes establecimos diferentes colaboraciones para impulsar el proyecto y sensibilizar a a entidades del tercer sector. Así mismo, llevamos a cabo también jornadas de sensibilización a la Inspección de Educación y a los equipos directivos como máximos responsables, estos últimos, del desarrollo del proyecto. $Y$ para finalizar, realizamos una sensibilización a los servicios educativos, quienes posteriormente actuarían como dinamizadores de la acción, estableciendo puentes entre centros y entidades y acompañando a los centros educativos en el proceso de desarrollo del proyecto.

\subsection{Formación específica}

Para los centros educativos y las entidades colaboradoras se planificó una acción formativa de cinco años, de acuerdo con el tiempo que el Decreto anteriormente mencionado- estipulaba para implementar de forma gradual el proyecto. La formación, de 15 horas, fue pensada para ser impartida conjuntamente a centros y entidades de una misma zona. El objetivo era facilitar un espacio de interacción entre las partes y generar la posibilidad que surgieran futuras iniciativas y proyectos. Pensamos también que esa formación debía ser impartida por profesionales del propio sistema 
educativo, en este caso, referentes de los servicios educativos. Ello nos permitiría empoderar a unos agentes que más tarde se convertirían en los dinamizadores del servicio comunitario en el territorio. Además, al no depender de formadores externos, facilitábamos la sostenibilidad del proyecto.

\subsection{Eficiencia y eficacia}

Como ya se ha comentado no hay innovación sin eficiencia demostrada, sin mejora en los objetivos que se persiguen. Por ello, a pesar de los buenos resultados obtenidos en la evaluación de la fase del pilotaje, decidimos llevar a cabo una evaluación anual durante todo el periodo de implementación (2015-2020) que nos permitiera conocer el grado de eficacia del propósito y reconducir, si era preciso, el proceso a partir de los resultados. Por otra parte, la eficacia también se debe valorar respecto al coste que conlleva. El servicio comunitario no podía comportar, para los centros educativos, un incremento significativo de esfuerzo, más allá de lo que requiere -a corto plazo- impulsar un nuevo proyecto. En este sentido muchos países han optado por contratar dinamizadores externos que faciliten esa coordinación centroentorno. En nuestro caso, preferimos incluir esas funciones en las propias de los servicios educativos. Las ventajas sobre las entidades externas es que los servicios educativos, por sus funciones, conocen el territorio y se relacionan con los diferentes agentes educativos de la zona. Ese aspecto facilitaba la acción y permitía al mismo tiempo, alinear el servico comunitario con otros proyectos existentes, optimizando los recursos y creando sinergias. Planes educativos de entorno, el Plan catalán de deporte en la escuela o las Escuelas verdes son algunos ejemplos.

\subsection{Sostenibilidad}

Como sabemos, la consolidación de un proyecto en el ámbito escolar es directamente proporcional a su capacidad de sostenerse en el tiempo. Para ello, era preciso ante todo, institucionalizar la acción, es decir, garantizar que el servicio comunitario formara parte del Proyecto Educativo de Centro y del proyecto curricular, y se concretara en el plan anual de centro. Con esa finalidad, la normativa que regula el servicio comunitario señala que debe formar parte de la programación curricular de una o varias materias de tercer y/o cuarto de ESO definidas en el plan anual de centro y que cada proyecto debe contener la vinculación curricular que lo fundamentaba. Así mismo, la misma normativa explicita que, a efectos de evaluación, el servicio comunitario tiene la consideración de proyecto transversal con incidencia en la calificación global de la materia, o materias, a la que esté vinculada.

Por otra parte, la sostenibilidad de un proyecto en red, como es el servicio comunitario, también depende de nuestra capacidad de fijar esas dinámicas de corresponsabilidad entre las partes. Hemos hablado ya de una figura dinamizadora estable que forma parte de los servicios educativos. Pero más allá de esa dinamización, hacía falta concretar una plataforma 
organizativa que sistematizase ese trabajo en red. Es decir, una plataforma con espacios participativos estables y periódicos, donde los diferentes agentes educativos: entidades, centros educativos, y administración local colaboraran tanto en la diagnósis de necesidades como en la búsqueda de entidades, en la propuesta de proyectos o en la valoración y visibilización de los mismos. Hay que subrayar aquí la relevancia de contar con la complicidad de la administración local como máxima conocedora del territorio, de sus necesidades y su capital social. En muchos municipios de Catalunya existen ya los Planes educativos de entorno que proporcionan esa plataforma organizativa. En ese marco resulta relativamente sencillo organizar grupos de trabajo con los objetivos mencionados. Pero para aquellos municipios que no tienen un plan educativo de entorno establecido, debíamos pensar en la posibilidad de elaborar un convenio entre el Departamento de Educación y la administración local. El objetivo era crear una comisión estable que garantizara el desarrollo, seguimiento y mejora del proyecto. Y así se acordó en la Comisión Mixta formada por la Federación de Municipios, la Asociación de Municipios y el Departamento de Educación.

\subsection{Capacidad de transferir}

La implantación, con carácter general, de un proyecto requiere garantizar que este pueda ser desarrollado por cualquier centro educativo. Para ello, elaboramos los modelos trasferibles a los que ya hemos hecho referencia. Estos permiten a los centros adaptar iniciativas documentadas a su contexto y necesidad. Los modelos transferibles pautan actividades didácticas y ofrecen recursos para trabajar con los alumnos el antes, el durante y el después de la realización del servicio, poniendo un énfasis especial en reforzar la correlación entre la diagnosis de necesidades reales, el análisis crítico y la implicación y compromiso activo en la mejora de esas necesidades. Confeccionamos, juntamente con las entidades pertinentes, transferibles de diferentes ámbitos (medio ambiente, intercambio intergeneracional, cooperación...). Todos los modelos pilotados y validados en la actualidadsiguen un mismo patrón. Comienzan con una fase de sensibilización, es necesario que los chicos y chicas encuentren sentido a movilizarse por algo, y esto requiere un tiempo para descubrir situaciones relevantes o experiencias existentes que les despierten ese deseo. Seguidamente, estos modelos, incorporan una fase de diagnosis y concreción del servicio, en el que ofrecen actividades para el alumnado de investigación y análisis crítico sobre el ámbito escogido para llevar a cabo. También ofrece pautas metodológicas para poder concretar propuestas de servicio que den respuesta a las necesidades detectadas y diseñar una evaluación que les permita, más tarde, conocer el grado de consecución de sus objetivos. En una tercera fase estos transferibles platean la ejecución del servicio. Aunque esta acción depende de las fases anteriores, el modelo ofrece orientaciones y recursos para fomentar 
la reflexión en la acción. Finalmente los transferibles aportan también actividades y recursos para acompañar el proceso de autoevaluación. Es importante tomar conciencia de lo que se ha hecho, de la importancia que ha tenido para cada participante en sus aprendizajes y de los valores y actitudes adquiridas.

\section{Estado de la situación y propuestas de futuro}

Transcurridos tres de los cinco años previstos para la implementación del servicio comunitario en todos los centros educativos de secundaria, podemos decir que el proceso se está desarrollando, en general, según las previsiones. El $62 \%$ de los centros educativos de secundaria han realizado proyectos de servicio comunitario durante el curso $2017 / 2018$ y se han formado un $82 \%$ de ellos. Así mismo disponemos de 202 convenios firmados con entidades colaboradoras y 114 convenios firmados con ayuntamientos con el objetivo de fomentar y facilitar la implementación del servicio comunitario en sus municipios.

En relación con los proyectos, el $67 \%$ de los centros educativos han llevado a cabo un solo proyecto de servicio comunitario, mientras que el $15 \%$ de los centros han realizado 4 o más proyectos, porcentaje que tiende a aumentar y que pone de manifiesto la gradualidad con que los centros implementan el proyecto. Por otra parte, mencionar que no hay una preferencia significativa a realizar el servicio comunitario en $3^{\circ}$ o $4^{\circ}$ de ESO. Así mismo comentar que el $62 \%$ de los proyectos se realizan en el ámbito de acompañamiento y apoyo a la escolarización (jóvenes guías, apoyo entre iguales, intercambio lingüístico...), en el ámbito del intercambio generacional (recuperación de la memoria histórica, iniciación a las tecnologías, acompañamiento a las personas mayores...) o en el de participación ciudadana (proyectos para potenciar la donación de sangre, evitar la discriminación, promover la cooperación...). Finalmente, mencionar que el $67 \%$ de los proyectos tienen un carácter transversal, aunque se mantiene la tendencia de ubicar el servicio comunitario principalmente en aquellas materias que tienen entre sus finalidades desarrollar la competencia social y ciudadana y la competencia comunicativa (Cultura y valores éticos, Lengua catalana y Educación éticocívica...)

En cuanto a la acción de los centros educativos, resaltar que el $83 \%$ de los centros han incluido el servicio comunitario en el Proyecto Educativo de Centro y el $83 \%$ en la Programación Anual de Centro. Así mismo el 92\% de los centros tienen en cuenta la evaluación del alumnado respecto el servicio comunitario en la calificación de las materias implicadas en el proyecto. Se observa en todos los porcentajes, una tendencia a aumentar.

En relación con el impacto del servicio comunitario sobre el aprendizaje del alumno, cabe comentar que el $98 \%$ de los centros consideran que el proyecto ha contribuido notablemente a la consecución de la competencia social y ciudadana, objetivo prioritario. Así 
mismo, un $71 \%$ de los centros consideran, en el mismo grado, que el servicio comunitario mejora el rendimiento académico en la materia o materias donde se ha desarrollado, hecho -como ya hemos comentado en la fase del pilotaje- que contribuye a visualizar la ventajas de utilizar métodos de aprendizaje des de la acción. Y finalmente, un $77 \%$ consideran que contribuye notablemente a la mejora de la convivencia en el centro. Este dato puede estar indicándonos que las metodologías colaborativas refuerzan aspectos como el conocimiento mutuo o la gestión de los conflictos entre el alumnado, pero también que el cambio de rol que ofrece a los alumnos el servicio comunitario, sacándolos de las aulas, proporciona nuevas oportunidades y rompe con las dinámicas conductuales establecidas.

Y por último, en relación con el impacto sobre el entorno: la realización de los proyectos ha generado nexos de colaboración y trabajo en red, con entidades locales en un $40 \%$ de los casos, con la administración local en un $21 \%$ de los casos y con otros centros educativos en un $19 \%$. Así mismo, el $18,35 \%$ del alumnado manifiesta su voluntad de continuar vinculado a la entidad en la que ha realizado el servicio, dato que contribuye a dar respuesta al objetivo de aumentar la participación del alumnado en el tejido asociativo del territorio.

Cuando comparamos estos resultados, con los obtenidos en el proceso de pilotaje -donde participaban centros especialmente motivados- observamos, en general, una mejora en los resultados. Eso, junto con la óptima valoración extraída de las últimas formaciones, nos hace pensar que entre todos y gracias especialmente al esfuerzo de los profesionales del territorio y los centros educativos, hemos logrado evitar aquellos riesgos que comportaba implementar una acción educativa con carácter obligatorio. Se han conseguido compartir las bondades de un proyecto $y$, entre todos, hemos contribuido a situar la competencia social y ciudadana en la primera línea de la práctica educativa.

Como propuesta de futuro, solo lanzar una reflexión. El servicio comunitario parece estar contribuyendo de forma eficaz y eficiente a desarrollar el espíritu crítico y aumentar la implicación y compromiso de nuestros jóvenes. Les ayuda a ver la ciudadanía más a allá de los derechos y a entenderla como una competencia que nos define. Si entendemos que eso forma parte de la finalidad educativa, que hemos de ser capaces de formar personas implicadas y comprometidas con su entorno ¿no deberíamos pensar en utilizar los beneficios que en materia de ciudadanía nos ha brindado el servicio comunitario y transferirlo a todas las etapas escolares?¿no deberíamos situar el servicio comunitario también como una acción curricular de carácter obligatorio en la educación primaria?

\section{Referencias bibliográficas}

Batlle; R (2013). El aprendizaje servició en España. El contagio de una 
revolución pedagògica necesaria.

Madrid: PPC.

Elzo, J. (2007). La educación del futuro y los valores. Debats d'Educació.

Barcelona: Universitat Oberta de Catalunya y Fundación Jaume Bofill.

Disponible en

https://www.youtube.com/watch? $v=p$

uy0mApiSg

Puig, J.; Batlle, R.; Bosch,C.; de la

Cerda, M.; Climent, T.; Gijón, M. ;

Graell, M.; Martín, X.; Muñoz, A.;

Palos, J.; Rubio, L.; Trilla,J. (2009).

Aprendizaje servicio (ApS). Educación y

compromiso cívico. Barcelona: Graó.

Terricabras, J. M. (2008). Súbdits o

ciutadans. Arxiu de l'Agenda

Llatinoamericana. Disponible en

http://www.mercaba.org/FICHAS/AlfaO

mega/297/alcoholismo.htm

Decreto 187/2015, de 25 de agosto,

d'ordenació dels ensenyaments de

l'educació secundària obligatòria. Art.

16 Servei Comunitari. Generalitat de

Catalunya.

Departament d'Ensenyament (2017).

Marc de la innovació pedagògica a

Catalunya. Generalitat de Catalunya. 